\title{
Исследование кинетических закономерностей твердофазного концентрирования ионов кальция природными сорбентами
}

\author{
® 2019 Сютова Е.А. ${ }^{1}$, Джигола Л.А. ${ }^{2}$ \\ ${ }^{1}$ ФГБОУ ВО «Астраханский государственный медицинский университет», Астрахань \\ ${ }^{2}$ ФББОУ ВО «Астраханский государственный университет», Астрахань \\ Поступила в редакцию 3.08.2019 г.
}

DOI: $10.17308 /$ sorpchrom.2020.20/2381

Для борьбы с зимней скользкостью широкое применение нашли комбинированные противогололедные смеси, имеющие в составе механическую часть из природных материалов и небольшие количества солей. В основе получения таких смесей лежит сорбционное насыщение различными модифицирующими компонентами, среди которых наиболее распространены хлориды натрия, калия, кальция и магния. Данное исследование посвящено определению оптимальных условий адсорбции ионов кальция природными сорбентами Астраханской области, а также оценке аппроксимаций химических и диффузионных моделей для описания кинетики сорбционного концентрирования с целью создания противогололедных смесей. Путем нехимической переработки природного сырья Астраханской области, добываемого открытым карьерным способом получены сорбенты: мергель, минерал опал мергелевого типа (опока), глина.

Кинетические исследования адсорбции ионов кальция проводили, оценивая убыль сорбата в различные промежутки времени методом прямой потенциометрией с ионселективным электродом марки «ЭЛИС-121Са» относительно хлорсеребряного электрода сравнения «ЭВЛ-1М3.1» с учетом фона. Исследование влияния среды на адсорбцию проводили с помощью комбинированного стеклянного электрода марки ЭСК-10601/7, что позволило установить максимальную ёмкость поглощения ионов кальция опалом мергелевого типа при $\mathrm{pH}=6.94$, мергелем при $\mathrm{pH}=5.61$ и при $\mathrm{pH}=6.45$ глиной.

Анализ изотерм адсорбции ионов $\mathrm{Ca}^{2+}$ при исследуемых температурах позволяет отметить характер процесса: на глине и мергеле - эндотермический; на опале мергелевого типа - экзотермический. Максимальной сорбционной емкостью к ионам $\mathrm{Ca}^{2+}$ обладает опал мергелевого типа, для глины и мергеля данные значения приблизительно одинаковы. Для выявления сорбатных взаимодействий, были вычислены изостерические теплоты адсорбции ионов $\mathrm{Ca}^{2+}$. Анализ характера изменения изостерических теплот адсорбции при различных величинах степени заполнения сорбента указывает на то, что изучаемые природные сорбенты имеют гетерогенную поверхность с центрами различной степени активности.

Интерпретация кинетических закономерностей адсорбции ионов кальция на природных сорбентах позволяет отметить, что поглощение ионов кальция протекает в смешаннодиффузионном режиме. Графическое нахождение и теоретический расчет: констант внешней и внутренней диффузии, значения критерия Био, кажущейся энергии активации позволяют сделать вывод, что определяющей в стадии лимитирования является внутренняя диффузия для мергеля и глины, внешняя - для опала мергелевого типа. Установлен вклад в общую скорость процесса стадии химического взаимодействия сорбата с поверхностью сорбентов, что подтверждается соответствием экспериментальных данных и кинетической модели Хо-Маккей. Экспериментально найденные количественные характеристики адсорбции ионов кальция, позволяют сделать вывод о возможности применения изучаемых сорбентов в качестве носителей для производства фрикционных противогололедных материалов. Процесс работы полученных противогололедных систем происходит за счет выделения энергии при совместном физико-химическим взаимодействии носителей и ионов кальция с поверхностью льда.

Ключевые слова: сорбционное концентрирование, хемосорбция, изотермы, внутренняя диффузия, внешняя диффузия, природные сорбенты. 


\section{Введение}

Для повышения безопасности движения транспортных средств в зимний сезон активно применяются комбинированные противогололедные смеси, состоящие из механической части, применяемой для увеличения сцепления (песок, щебень), и химических соединений (хлориды, сульфаты, ацетаты). Механические воздействия интенсивно движущегося транспорта при использовании противогололедных смесей приводят к попаданию используемых реагентов в окружающую экосистему. Данный антропогенный фактор вызывает изменения состава основных компонентов окружающей среды и деградацию обитающих в них организмов.

Преимуществом использования природных материалов в качестве механической части (носителя) для противогололедных смесей является высокая селективность, химическая, термическая и механическая стабильность, экологичность и экономичность, однако имеется ограничение по температурным интервалам их применимости. По физико-химическим свойствам и антропогенным показателям, наиболее пригодны для борьбы с зимней скользкостью хлориды натрия, калия, кальция и магния [1]; в качестве модифицирующего компонента оптимальным выбором является хлорид кальция [2]. Широкое применение нашли именно противогололедные материалы, содержащие соли кальция, марок АЙСМЕЛТ (ICEMELT), Bionord Universal, Ecosol (Россия) и ICEHIT LITE MIX, DMS-SW GULF STREAM TYPE (Европа).

В работах [3-5] авторами указывается возможность применения материалов, добываемых открытым способом на территории Астраханской области, опоки мергелевого типа, мергеля и глины при производстве противогололедных смесей. Физические показатели (особенности строения, цветность), экологичность, низкая себестоимость, в совокупности с высокой долей солености самих материалов позволяет применять их в качестве механической части противогололедных смесей. В работах авторов $[6,7]$ показано применение данных материалов для удерживания и концентрирования токсичных соединений различной природы.

Сорбционное насыщение различных модифицирующих компонентов лежит в основе многих технологических процессов получения материалов с определенными физико-химическими свойствами [8]. С целью разработки технологии и химикотехнологическом обосновании получения сорбционных материалов с заданными свойствами необходимо оптимизировать процессы, протекающие в динамических условиях, что, в свою очередь, определяется природой и структурой материала носителя, и их взаимодействием с сорбируемым компонентом.

Адсорбционная ёмкость природных сорбентов и необходимое время контакта являются ключевыми показателями, которые используются при подборе адсорбентов, определяемыми равновесием и кинетикой адсорбции. Статическая адсорбция может предсказать только конечное равновесное состояние системы. Кинетический анализ позволяет установить скорость поглощения адсорбата и время, необходимое для завершения процесса адсорбции. Вследствие сложности и многостадийности сорбционного процесса поглощения ионов металлов из водных растворов, для выявления лимитирующей стадии кинетики целесообразно оценить адекватность нескольких кинетических моделей. Таким образом, использование кинетических моделей при описании сорбционных процессов поглощения ионов кальция может позволить охарактеризовать механизм сорбции природными сорбентами и установить лимитирующую стадию адсорбции для последующего определения рациональных условий осуществления технологического процесса.

Целью настоящей работы является выбор оптимальных условий адсорбции ионов кальция природными сорбентами Астраханской области, а также оценка ап- 
проксимаций химических и диффузионных моделей для описания кинетики сорбционного концентрирования.

\section{Эксперимент}

Объектами исследований являлись сорбенты, полученные путем нехимической переработки минерального сырья Астраханской области, добываемого открытым карьерным способом: мергель, минерал опал мергелевого типа (опока), глина. Для получения сорбентов сырьё из карьеров подвергается размалыванию на шаровых мельницах, далее на виброситах отсеиваются частицы с заданным размером. Следующим этапом является промывание в воде с целью освобождения от растворимых компонентов и высушивание до постоянной массы. Природные сорбенты использовали в воздушно-сухом состоянии. Степень раздробленности природных сорбентов соответствует для минералов мергеля и опал мергелевого типа -0.1 см, глины -0.05 см.

Для анализа содержания ионов кальция применятся множество методов: гравиметрические, титриметрические, спектрофотометрические, капиллярного электрофореза, фотометрические и атомно-эмиссионные. Электрохимические методы способны обеспечить определение огромного числа как неорганических, так и органических веществ. Для них характерна высокая чувствительность и селективность, быстрота отклика на изменение состава анализируемого объекта, легкость автоматизации и возможность дистанционного управления, они не требуют дорогостоящего оборудования и могут применяться в лабораторных, производственных и полевых условиях. В отличие от других методов анализа, позволяющих оценить лишь общую концентрацию вещества, ионометрия позволяет оценить активность свободных ионов и поэтому играет большую роль в изучении распределения ионов между их различными химическими формами [9-10].

Содержание ионов кальция до и после адсорбции проводили методом прямой потенциометрии с учетом фона. Измеряли потенциал ИСЭ на ионы кальция «ЭЛИС$121 \mathrm{Ca}$ относительно хлорсеребряного электрода сравнения «ЭВЛ-1М3.1» анализатором жидкости «ЭКСПЕРТ-001-3». Исследование влияния рН на адсорбцию проводили с помощью комбинированного стеклянного электрода марки ЭСК-10601/7. Установлено, что максимальное значение ёмкости поглощения достигается при $\mathrm{pH}=6.94$ опалом мергелевого типа, при $\mathrm{pH}=5.61$ мергелем, при $\mathrm{pH}=6.45$ глиной. Модельные растворы хлорида кальция готовили объёмно-весовым способом, рабочие растворы заданной концентрации - методом последовательного разбавления. Соотношение водный раствор $\mathrm{CaCl}_{2}$ : сорбент поддерживали $25: 1\left(\mathrm{~cm}^{3} / \Gamma\right)$. Кинетические исследования адсорбции ионов кальция проводили, оценивая убыль сорбата в различные промежутки времени, при постоянном перемешивании.

Количество сорбированного вещества $Q$ (ммоль/г) рассчитывали по формуле:

$$
Q=\frac{\left(C_{0}-C_{\tau}\right) \cdot V}{m} \cdot 1000
$$

где $C_{0}-$ начальная концентрация $\mathrm{Ca}^{2+}$ в растворе $\left(C_{0}=10\right.$ ммоль/дм $\left.{ }^{3}\right)$; $C_{\tau}$ - равновесная концентрация $\mathrm{Ca}^{2+}$ (ммоль/дм $\left.{ }^{3}\right) ; V$ - объём раствора в дм ${ }^{3}$; $m$ - масса навески воздушно-сухого минерального сорбента, г.

По полученным данным определяли степень завершенности процесса $(F)$ по формуле:

$$
F=Q_{\tau} / Q_{\mathrm{e}}
$$

где $Q_{\text {е и }} Q_{\tau}$ - количество сорбированного вещества на единицу массы сорбента в состоянии равновесия и в момент времени $\tau$, соответственно (ммоль/г). 
С целью оценки вклада сорбатных взаимодействий рассчитывали дифференциальные теплоты адсорбции по тангенсу угла наклона изостер. Метод расчета теплот адсорбции по изостерам основан на уравнении Клайперона-Клаузиса:

$$
\ln C=-q_{s t} / R T+B
$$

где $C$ - концентрация сорбтива в растворе, $\left(\right.$ ммоль/дм $\left.{ }^{3}\right) ; T$ - температура, К; $R$ - универсальная газовая постоянная, Дж/моль $\cdot \mathrm{K} ; q_{s t}-$ изостерическая теплота перехода сорбтива в сорбент, Дж/моль; $B$ - постоянная интегрирования.

Для определения лимитирующей стадии исследуемых сорбционных процессов, полученные кинетические кривые сорбции представляли в координатах уравнения Бойда-Адамсона, которое возможно применять для случая внешней и внутренней диффузии в зависимости от допущений:

$$
F=1-\frac{6}{\pi^{2}} \sum_{n=1}^{\infty} \frac{1}{n^{2}} \exp \left(-D n^{2} \pi^{2} / r_{0}^{2}\right)
$$

$F$ - степень завершенности процесса, зависит только от размера зерна $r_{0}$, эффективного коэффициента взаимодиффузии и является функцией параметра $D \tau / r_{0}^{2}$; $D$ - эффективный коэффициент диффузии вещества внутри зерна сорбента, $\mathrm{cm}^{2} / \mathrm{c}$; $\tau$ - время, в течение которого достигается данная величина $F ; n$ - число слагаемых целые числа $1,2,3, \ldots ; r_{0}$ - средний радиус зерна сорбента, см; отношение $\mathrm{B}=D \pi^{2} / r_{0}^{2}-$ кинетический коэффициент; $Q_{\mathrm{e}}$ и $Q_{\tau}-$ количество сорбированного вещества на единицу массы сорбента в состоянии равновесия и в момент времени $\tau$, соответственно (ммоль/г).

После подстановки кинетического коэффициента в уравнение (4) зависимость принимает следующий вид:

$$
\mathrm{F}=1-\frac{6}{\pi^{2}} \sum_{\mathrm{n}=1}^{\mathrm{n}=\infty} \frac{1}{\mathrm{n}^{2}} \exp \left(-\mathrm{B} \tau \cdot \mathrm{n}^{2}\right)
$$

$B \tau$ - безразмерный параметр, предложенный Бойдом. Представляет собой решение задачи диффузии в твердом теле определенной формы. Величины $B \tau$ рассчитаны для любых значений степени завершенности процесса $(F)$ и сведены в таблицы как $B \tau=f(F)$ [11]. Коэффициент диффузии после нахождения значения $B \tau$ для определенной степени завершенности процесса, рассчитывался по уравнению:

$$
D=\left(B \tau \cdot r^{2}\right) /\left(\pi^{2} \cdot \tau\right)
$$

Преобладание внешнедиффузионного механизма или кинетики адсорбции характеризуется линейной зависимостью в координатах «-ln $(1-F)=f(\tau)$ ».

Уравнение для малых степеней заполнения поверхности сорбентов, то есть уравнение Бойда-Адамсона в линейной форме представляет собой решение задачи диффузии в твердом теле определенной формы и применимо для конечных стадий внутренней диффузии, это другая форма уравнения (5) где $n=1$ :

$$
\ln (1-F)=-\gamma \cdot \tau+A
$$

При пленочном механизме диффузии множитель перед $\tau$ в уравнении (7) равен тангенсу угла наклона прямой к оси абсцисс и позволяет рассчитать константу скорости внешней диффузии $\left(\mathrm{D}_{6 н}, \mathrm{c}^{-1}\right)$ :

$$
\gamma=\frac{3 D_{6 н} C^{0}}{r_{0} \delta C_{\text {погл }}}
$$

где $D_{6 н}-$ коэффициент диффузии вещества через пленку жидкости толщиной $\delta$, покрывающую зерно сорбента (значение которой принималось равным $5 \cdot 10^{-3} \mathrm{~cm}$ ) [12]; $C^{0}$ и $C_{\text {погл }}$ - концентрация сорбтива в растворе и сорбенте, ммоль/дм ${ }^{3} ; r_{0}-$ средний радиус зерна сорбента, см. 
Поскольку внешнедиффузионная кинетика описывает только начальный этап установления равновесия в рассматриваемых системах, то для того, чтобы оценить вклад внутренней диффузии в процесс адсорбции использовали асимптотическое приближение зависимости «F $=f(\tau) »$ в начальный момент при малых степенях заполнения сорбента, подчиняющееся закону корня:

$$
F=k_{d} \cdot \tau^{1 / 2}
$$

где $k_{d}-$ константа скорости внутренней диффузии, ммоль $\Gamma^{-1} \cdot$ мин $^{-1 / 2} ; \tau-$ время, мин.

В большинстве случаев [12] зависимость « $F=f\left(\tau^{1 / 2}\right) \gg$ является мультилинейной, описывается уравнением (10), не выходит из начала координат и характеризуется несколькими участками:

$$
F=k_{d} \cdot \tau^{1 / 2}+A
$$

где $A$ - отрезок, отсекаемый на зависимости «F=f( $\left.\tau^{1 / 2}\right) »$, на оси ординат, и равный толщине граничного слоя, а $k_{d}$ - угловой коэффициент линейного участка. Величины коэффициента $A$ во многих случаях соизмеримы с погрешностью их определения. Отрицательные и близкие к нулю значения $A$ предполагают, что толщина пленки мала и ее влияние на процесс диффузии минимально.

Для подтверждения лимитирующей стадии поглощения ионов кальция изучаемыми сорбентами рассчитана кажущаяся энергия активации суммарного процесса сорбции ионов кальция (табл.2). Энергия активации процесса находилась из зависимости $\ll \ln k_{d}=f(1 / \tau) »$, которая является уравнением прямой:

$$
\lg k_{d}=\lg k_{d 0}-E_{a} / R T
$$

где $k_{d}$ и $k_{d 0}$ - константы скорости внутренней диффузии в момент времени $\tau$ и $\tau_{0}$ соответственно, ммоль $\Gamma^{-1} \cdot$ мин $^{-1 / 2} ; E_{a}-$ кажущаяся энергия активации процесса диффузии, Дж/моль $\cdot$ К.

Кинетический режим диффузионного процесса определяется численным значением критерия Био $\left(B_{i}\right)[13]$, который определяли по уравнению:

$$
B_{i}=\frac{D_{6 H} r}{D \delta K_{p}}
$$

где $K_{\mathrm{p}}$ - это константа адсорбционного равновесия, определяемая как $K_{\mathrm{p}}=Q_{\tau} / C_{\tau}$; $D_{6 н}-$ коэффициент диффузии вещества через пленку жидкости толщиной $\delta$, покрывающую зерно сорбента (значение которой принималось равным $5 \cdot 10^{-3} \mathrm{~cm}$ ) $; r$ - размера зерна сорбента, см; $D$ - эффективный коэффициент внутренней диффузии, $\mathrm{cm}^{2} / \mathrm{c}$.

Как отмечается в [14], если $B_{i}>20$ то лимитирующая стадия диффузионного процесса протекает во внутридиффузионной области, если $B_{i}<1$ то внешнедиффузионной, промежуточные значения свидетельствуют о смешанной кинетике процесса.

Для оценки лимитирующей стадии определены энергетические характеристики взаимодействий. Энергию активации $\left(E_{a}\right)$ находили графически, используя уравнение Аррениуса в линейной форме:

$$
\ln k_{2}=\ln p z_{0}-E_{a} / R T
$$

где $k_{2}$ - константы скорости, полученные для уравнений псевдо-второго порядка, $\Gamma \cdot(\text { ммоль } \cdot \text { мин })^{-1} ; p z_{0}$ - предэкспоненциальный фактор в уравнении Аррениуса; $R$ - универсальная газовая постоянная, Дж/моль·К; $T$ - температура, К.

Графически найденные постоянные уравнения Аррениуса, применяли для определения энтропии активации $\Delta \mathrm{S}^{\#}$ :

$$
\lg p z_{0}=10,36+\lg T+\Delta S * / 2,3 R
$$

Для оценки вклада химической стадии в общую скорость адсорбции рассматривали применимость формальных уравнений кинетики. Модель Лагергрена, описы- 
вающая скорость псевдо-первого порядка, основана на определении изменения сорбционной емкости в гетерогенной смеси может быть представлено в виде:

$$
\frac{d Q_{\tau}}{d \tau}=k_{1}\left(Q_{e}-Q_{\tau}\right)
$$

где $k_{1}$ - константа скорости псевдо-первого порядка $\left(\right.$ мин $\left.^{-1}\right) ; Q_{\mathrm{e}}$ и $Q_{\tau}-$ количество сорбтива на 1 г сорбента в равновесном состоянии и при времени $\tau$, соответственно (ммоль/г). Уравнение при интегрировании с граничными условиями от $\tau=0$ до $\tau=\tau$ и $Q_{\tau}=0$ до $Q_{\tau}=Q_{\tau}$ принимает следующий вид:

$$
\ln \left(Q_{e}-Q_{\tau}\right)=\ln \left(Q_{e}\right)-k_{1} \tau
$$

Для проведения анализа экспериментальных данных с позиции модели Лагергрена необходимо построение кинетических кривых в координатах $\ll-\ln \left(1-Q_{\tau}\right)=f(\tau) »($ рис. 5$)$.

Для описания вклада «сорбат-сорбатных» взаимодействий при адсорбции ионов кальция на минеральных сорбентах в скорость процесса применялась модель Хо и Маккей отражающая взаимодействие для реакции псевдо-второго порядка. В интегральной форме классическое уравнение скорости псевдо-второго порядка имеет вид [15]:

$$
Q_{\tau}=\frac{\tau}{\frac{1}{k_{2} \cdot Q_{e}^{2}}+\frac{\tau}{Q_{e}}}
$$

где $k_{2}$ - константа скорости сорбции в рамках применяемой модели, г.(ммоль·мин) ${ }^{-1}$; $\tau$ - время, мин.

При линеаризации уравнения (18) минимизируется вклад эффективного параметра $Q_{e}$, что позволяет избежать проблемы, связанной с его достижением:

$$
\frac{\tau}{Q_{\tau}}=\frac{1}{k_{2} \cdot Q_{e}^{2}}+\frac{1}{Q_{e}} \tau
$$

Данное уравнение (19) позволяет представить полученные эксперименталь-

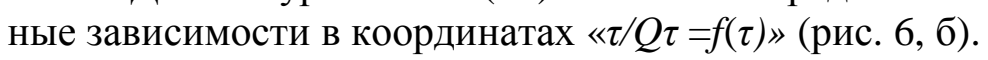

\section{Обсуждение результатов}

Опока мергелевого типа и мергель относятся к мезопористым материалам и представляют класс монтморилонитов, для которых характерным является содержание $\mathrm{SiO}_{2}$ в пределах $30 \%$ и наличие около $3 \%$ оксидов $\mathrm{Al}_{2} \mathrm{O}_{3}$ и $\mathrm{Fe}_{2} \mathrm{O}_{3}$, что свидетельствует о глинистой составляющей породы. Глина содержит около $70 \% \mathrm{SiO}_{2}$ и $12 \%$ $\mathrm{Al}_{2} \mathrm{O}_{3}$. Остальное количество приходится на оксиды и хлориды $\mathrm{Na}, \mathrm{Mg}, \mathrm{Ca}, \mathrm{K}$ и др. металлов. Мергель и опока имеют близкие качественные и количественные характеристики, но обладают разной пространственной структурой. У опоки мергелевого типа наблюдается хлопьевидно-глобулярная микроструктура, для мергеля характерна слоистая структура, как и для глины Астраханского месторождения, что подтверждается его способностью к набуханию [16].

На рис. 1 представлены изотермы адсорбции ионов кальция исследуемыми природными сорбентами в виде зависимости количества, поглощённого сорбентом иона кальция $\left(Q\right.$, ммоль/г) от равновесной концентрации $\left([C]\right.$, ммоль/дм $\left.{ }^{3}\right)$ при температурах $275 \pm 2$ К, $293 \pm 2$ К и $318 \pm 2$ К.

Наибольшая сорбционная емкость по ионам кальция характерна для опала мергелевого типа $(Q=3.5$ ммоль/г), для глины и мергеля данные значения приблизительно одинаковы $(Q=2.5$ ммоль/г). На рис. 1а величины количества поглощенного 
вещества $(Q)$ при пониженном значении температуры значительно выше, чем при комнатной и повышенной, соответственно. На рис. 1б и 1в наблюдается обратная зависимость. На всех кривых можно выделить три участка, начальный линейный участок описывается изотермой Генри, и в данном диапазоне концентраций сорбата поглощение идет по более доступным активным центрам. Наблюдаемые перегибы связаны с изменением моделей сорбционного равновесия и вероятно, заполнение активных центров поверхности сорбентов происходит последовательно. Выход всех кривых на плато указывает на насыщение сорбентов ионами кальция при исследуемых температурах. Анализ изотерм адсорбции при исследуемых температурах (рис 1.) позволяет отметить характер процесса: на глине и мергеле - эндотермический; на опале мергелевого типа - экзотермический, что свидетельствует о различии в механизмах поглощения ионов кальция.

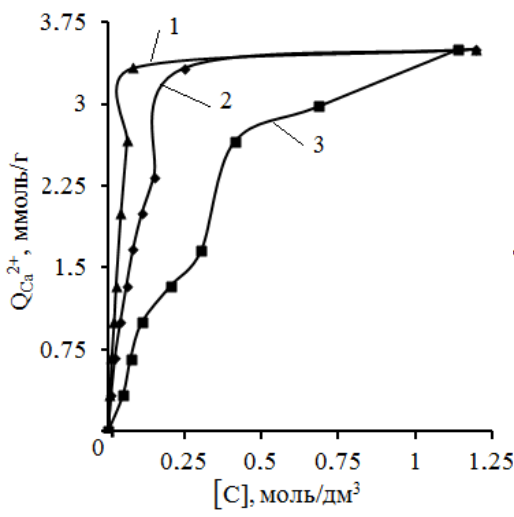

a

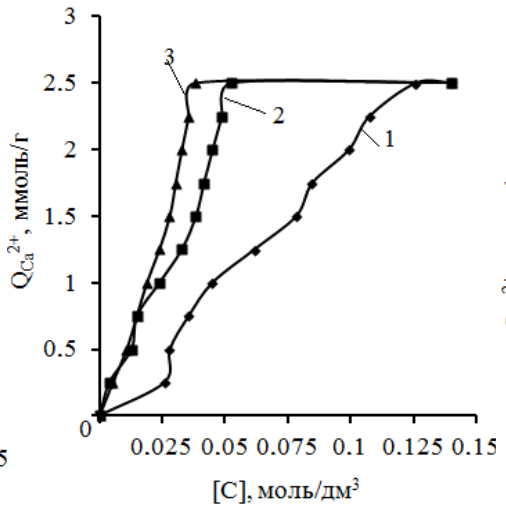

6

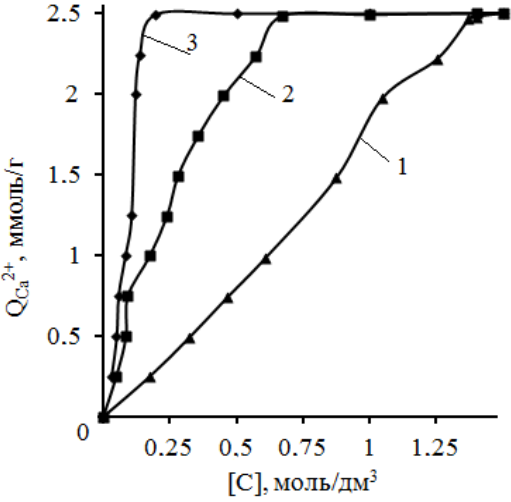

B

Рис. 1. Изотермы адсорбции ионов кальция на природных сорбентах:

a - опал мергелевого типа, б - глина, в - мергель; при температуре растворов: $1-275 \pm 2$ К, $2-293 \pm 2$ К и $3-318 \pm 2 \mathrm{~K}\left(\mathrm{t}_{\mathrm{p}}=0.95\right)$

Анализируя полученные результаты, можно предположить, что увеличение адсорбции ионов кальция с изменением температуры, скорее всего, связано с увеличением растворимости, а также с изменением заряда поверхности [17] и, возможно, за счет взаимодействия ионов кальция с активными центрами природных сорбентов мергеля и глины, и конкуренцией с молекулами растворителя за активные центры. Для выявления сорбатных взаимодействий, используя изотермы сорбции, полученные при исследуемых температурах, были вычислены изостерические теплоты адсорбции ионов $\mathrm{Ca}^{2+}[18]$ на изучаемых природных сорбентах, которые приведены в таблице 1.

Таблица 1. Изостерические теплоты адсорбции $\left(q_{s t}\right)$ ионов $\mathrm{Ca}^{2+}$ на природных сорбентах при разных величинах степени заполнения сорбента $(F)$

\begin{tabular}{|c|c|c|c|c|c|}
\hline \multicolumn{2}{|c|}{ Глина } & \multicolumn{2}{c|}{ Опал мергелевого типа } & \multicolumn{2}{c|}{ Мергель } \\
\hline$F$ & $q_{s t}$ & $F$ & $q_{s t}$ & $F$ & $q_{s t}$ \\
\hline 0.1 & 31.65 & 0.1 & 29.58 & 0.1 & 0.662 \\
\hline 0.2 & 12.83 & 0.2 & 31.85 & 0.2 & 17.92 \\
\hline 0.5 & 10.53 & 0.5 & 35.68 & 0.5 & 23.01 \\
\hline 0.9 & 12.64 & 0.9 & 37.15 & 0.9 & 29.36 \\
\hline
\end{tabular}

Разница в значениях изостерических теплот адсорбции при различных величинах степени заполнения сорбента указывает на то, что изучаемые природные сорбенты имеют гетерогенную поверхность с центрами различной степени активности. Вероятно, на начальных стадиях адсорбция ионов кальция имеет место на самых ак- 
тивных центрах (кислотных центрах Льюиса), с увеличением величины степени заполнения сорбента - на местах с меньшей активностью (основных центров Бренстеда), поэтому ожидаемым является уменьшение величин изостерических теплот адсорбции с ростом степени заполненности сорбента, что и наблюдалось в случае адсорбции на глине. Увеличение значений изостерической теплоты адсорбции в случае поглощения на опале мергелевого типа и мергеле объясняется усилением вклада энергии взаимодействия в системе «сорбат-растворитель-сорбент» в общую энергию адсорбции при увеличении плотности сорбционного слоя. Взаимодействие, характеризующееся изостерической теплотой адсорбции в системе «сорбат-растворительсорбент» появляется даже при малых величинах степени заполненности сорбента и это указывает на то, что заполнение происходит неравномерно и задолго до насыщения сорбента сорбатом начинается заполнения тонких пор. По увеличению величин теплот адсорбции ионов кальция природные сорбенты можно расположить в ряд:

глина < мергель < опал мергелевого типа.

На рисунке 2 представлены интегральные кинетические кривые сорбции ионов кальция исследуемыми природными сорбентами в виде зависимости количества, поглощённого сорбентом ионов кальция $(Q$, ммоль/г), от времени $(\tau$, сек) при исследуемых температурах.

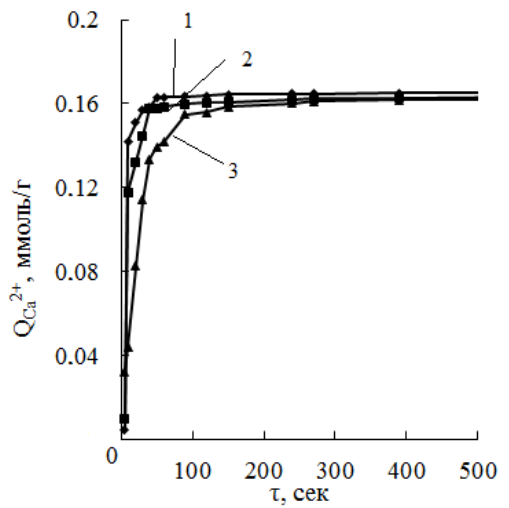

a

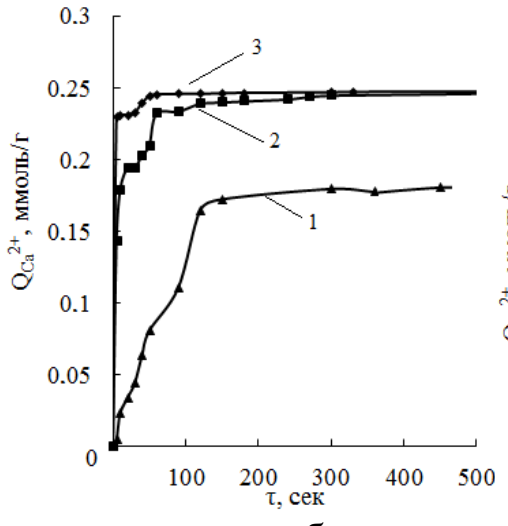

6

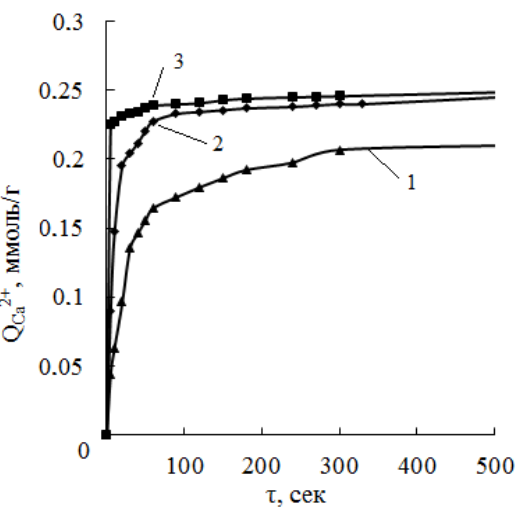

B

Рис. 2. Интегральные кинетические кривые адсорбции ионов кальция на минеральных сорбентах: а) опал мергелевого типа, б) глине, в) мергеле.

$$
\left(\mathrm{C}_{\mathrm{Ca}}^{0}{ }^{2+}=10^{-3} \text { моль/дм }{ }^{3}, \mathrm{~V}=50 \mathrm{~cm}^{3}, \mathrm{~m}=5\right. \text { г) }
$$

Анализ кинетических кривых (рис.2) позволяет отметить аналогичную температурную зависимость при поглощении ионов кальция во времени, как и при исследовании изотерм адсорбции (рис.1). Выход интегральных кинетических кривых на плато за 2-3 минуты свидетельствует об эффективном поглощении ионов кальция, при этом степень поглощения составляет 98\% от начального содержания сорбата. Адсорбция мергелем протекает медленнее, время достижения равновесия составляет 3-5 минут при этом поглощается $85 \%$ сорбата.

С целью определения лимитирующей стадии процесса адсорбции ионов кальция на природных сорбентах экспериментальные данные были обработаны по уравнению Бойда-Адамсона [11], которое применимо для случая внешней и внутренней диффузии в зависимости от допущений. Для подтверждения вклада кинетического режима в лимитирующую стадию поглощения ионов кальция изучаемыми сорбентами рассчитана кажущаяся энергия активации. Кинетический режим диффузионного процесса характеризуется численным значением критерия Био $\left(B_{i}\right)$, по величинам которых можно предположить лимитирующую стадию области диффузионного процесса [19]. 
На основании полученных расчетных данных построены зависимости «-ln$(1-F)=f(\tau) »$ при малых степенях заполнения сорбента (рис. 3).

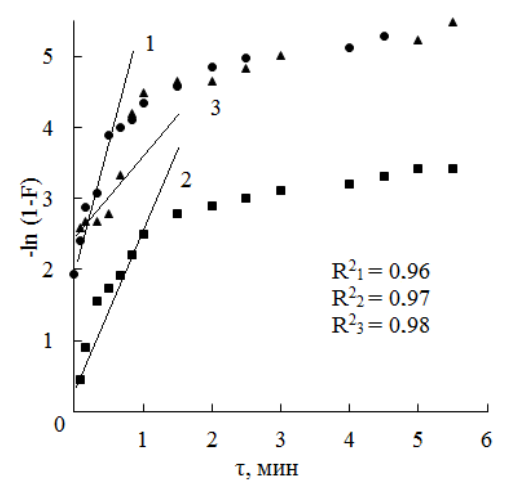

Рис. 3. Зависимость «- $\ln (1-F)=f(\tau) »$ в системах $\ll \mathrm{Ca}^{2+}-$ сорбент»:

1 - опал мергелевого типа; 2 - мергель;

3 - глина для случая внешней диффузии при температуре $293 \pm 2$ K

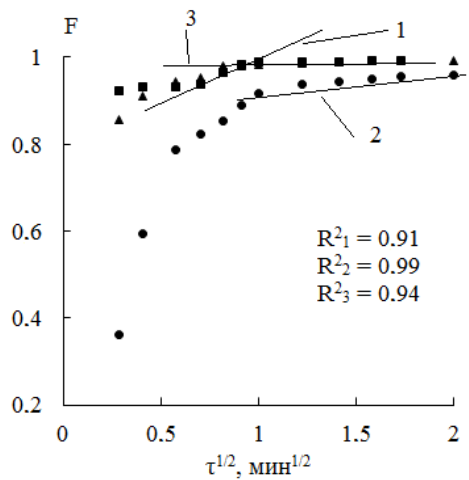

Рис. 4. Зависимость $F=f\left(\tau^{1 / 2}\right)$ в системах « $\mathrm{Ca}^{2+}$ - сорбент»: 1 - опал мергеле-

вого типа; 2 - мергель; 3 - глина, для случая внутренней диффузии при температуре $293 \pm 2 \mathrm{~K}$

Анализ полученных зависимостей (рис. 3) позволяет отметить, что только в первую минуту на начальных участках функция $-\ll \ln (1-F)=f(\tau)$ для исследуемых сорбционных процессов является линейной и аппроксимируются с уравнением (7). Наблюдаются высокие значения коэффициентов детерминации $\left(R^{2}\right)$ для линейных участков в данном временном интервале. Следовательно, в начальный момент процесса адсорбции ионов кальция, преобладает внешнедиффузионный механизм, так как при увеличении степени заполненности поверхности сорбента линейная зависимость не соблюдается после одной минуты. При возрастании степени завершенности процесса влияние внешнедиффузионного фактора уменьшается, а внутридиффузионного возрастает. На рис. 4 представлены зависимости « $F=f\left(\tau^{1 / 2}\right) »$ при температуре растворов $293 \pm 2$ К.

Полученные зависимости мультилинейны: начальный линейный участок описывает диффузию сорбата через слой раствора к поверхности сорбента, а второй линейный участок, из наклона которого определяется константа скорости внутренней диффузии $\left(k_{d}\right)$, описывает, собственно, внутридиффузионный процесс. Как отмечают многие авторы [13, 19-20] при внутренней (гелевой) диффузии кинетическая зависимость $\ll F=f\left(\tau^{1 / 2}\right) »$ должна выражаться прямой, которая при малых значениях $\tau$ имеет линейных характер, а затем искривляется, что наблюдается на рис 4. Линейность данных зависимостей в системе $« F=f\left(\tau^{1 / 2}\right) »$ при степени заполнения поверхности сорбента $F \rightarrow \infty$, означает, что лимитирование сорбционного процесса обусловлено внутридиффузионным переносом в данном интервале времени, поэтому преобразованное уравнение (10) можно использовать для расчета константы скорости внутренней диффузии ионов кальция в сорбентах. Результаты расчетов представлены в табл.2. Для установления влияния внутридиффузионных ограничений на скорость сорбционных процессов полученные в работе кинетические кривые также представляли в виде функции «B $\tau=f(\tau) »$ рассчитанные по уравнению (6) во всем диапазоне степени заполнения поверхности сорбента (рис. 5).

Полученные зависимости в координатах $\quad\left\langle B \tau=f(\tau) », \quad « F=f\left(\tau^{1 / 2}\right) » \quad\right.$ и $\ll-\ln (1-F)=f(\tau) »$ характеризуются близкими значениями коэффициентов детерминации, что свидетельствует о смешанно диффузионном характере лимитирующей стадии общей скорости сорбции. 


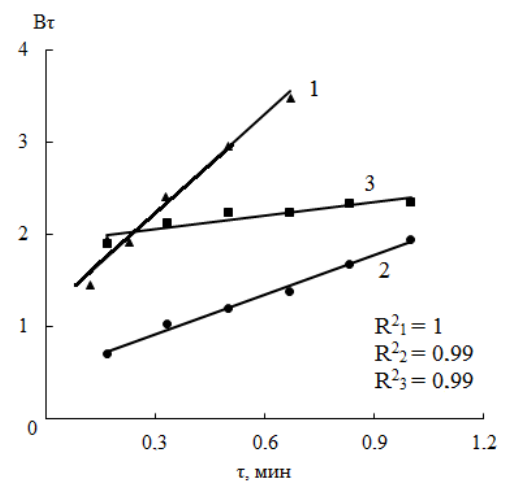

Рис. 5. Зависимость $B \tau=f(\tau)$ в системах « $\mathrm{Ca}^{2+}-$ сорбент»:

1 - опал мергелевого типа; 2 - мергель; 3 - глина, для случая внутренней диффузии при температуре $293 \pm 2 \mathrm{~K}$

Аналогично обрабатывались кинетические данные при температурах 275 и 318 К, что позволяет отметить сокращение времени достижения равновесия при повышении температуры, вероятно обусловленное снижением сопротивления внешней среды. Полученные результаты обработки интегральных кинетических кривых при степенях заполнения поверхности $(F)$ : мергель $F \leq 0.71$, глина $F \leq 0.82$ и опал мергелевого типа $F \leq 0.96$, представлены в таблице 2 .

Таблица 2. Кинетические параметры диффузионной кинетики ионов $\mathrm{Ca}^{+2}$ на природных сорбентах

\begin{tabular}{|c|c|c|c|c|c|c|c|c|c|c|}
\hline \multirow{2}{*}{\multicolumn{2}{|c|}{$\begin{array}{c}\text { Диффузионные } \\
\text { параметры }\end{array}$}} & \multicolumn{3}{|c|}{ Мергель } & \multicolumn{3}{|c|}{ Глина } & \multicolumn{3}{|c|}{ Опал мергелевого типа } \\
\hline & & 275 & 293 & 318 & 275 & 293 & 318 & 275 & 293 & 318 \\
\hline \multicolumn{2}{|c|}{$D_{\theta H^{\prime}} 10^{3}, \mathrm{~cm}^{2} / \mathrm{Mин}$} & 0.353 & 1.055 & 3.280 & 0.036 & 1.11 & 1.16 & 18.06 & 16.42 & 11.74 \\
\hline \multicolumn{2}{|c|}{$\begin{array}{c}k_{d} \cdot 10^{3}, \\
\text { ммоль } \cdot \Gamma^{-1} \cdot \text { Мин }^{-1 / 2}\end{array}$} & 210 & 229 & 277 & 4.60 & 25.91 & 39.54 & 13.8 & 10.9 & 9.6 \\
\hline \multicolumn{2}{|c|}{$D \cdot 10^{3}, \mathrm{~cm}^{2} /$ мин } & 0.13 & 0.14 & 0.15 & 7.52 & 8.65 & 7.98 & 0.63 & 0.23 & 0.09 \\
\hline \multicolumn{2}{|c|}{$B_{i}$} & 294 & 275 & 139 & 86 & 50 & 8 & 0.0018 & 0.0020 & 0.0022 \\
\hline \multirow{3}{*}{$\begin{array}{c}\text { Энергия } \\
\text { активации, } \\
\text { кДж/моль }\end{array}$} & $\begin{array}{l}E a \\
D B H\end{array}$ & \multicolumn{3}{|c|}{ не описывается } & \multicolumn{3}{|c|}{ не описывается } & \multicolumn{3}{|c|}{7.28} \\
\hline & $\underset{k d}{E a}$ & \multicolumn{3}{|c|}{4.08} & \multicolumn{3}{|c|}{36.34} & \multicolumn{3}{|c|}{ не описывается } \\
\hline & $\underset{D}{E a}$ & \multicolumn{3}{|c|}{2.42} & \multicolumn{3}{|c|}{0.78} & \multicolumn{3}{|c|}{33.11} \\
\hline
\end{tabular}

Значения коэффициентов внешней и внутренней диффузии, а также значения критерия Био $\left(B_{i}\right)$ свидетельствуют о смешанном диффузионном механизме адсорбции с преобладанием внутридиффузионных ограничений для мергеля и глины и внешнедиффузионных для опала мергелевого типа, в линейной области изотерм адсорбции, для которых были проведены кинетические исследования.

Величина кажущейся энергии активации, вычисленная по эффективным коэффициентам диффузии $(D)$ ионов кальция из водных растворов к активным центрам природных сорбентов уменьшается в ряду: опал мергелевого типа, мергель, глина. Диапазон значений для глины и мергеля - подтверждает большой вклад внутридиффузионного механизма, а для опала мергелевого типа - смешено-диффузионный механизм. Найденные значения энергии активации процесса в зависимости от преобладания различных стадий в механизм сорбции $\left(E_{a}{ }^{\text {вн }}\right.$ и $\left.E_{a}{ }^{\mathrm{kd}}\right)$ подтверждают полученные результаты. Полученные значения критерия Био у мергеля и глины уменьшающиеся с ростом температуры подтверждают, что лимитирующая стадия диффу- 
зионного процесса протекает во внутридиффузионной области, а для опала мергелевого типа следовательно предпочтительное протекание процесса во внешнедиффузионной области.

Большие ионы при диффузии вглубь сорбента частично замещают значительное количество воды находящейся в порах сорбента. При этом возрастает работа образования пустот в диффузионной среде. Все эти факторы увеличивают энергию активации и тем самым уменьшают скорость ионного обмена.

Одной из причин неустойчивости параметров диффузионных моделей может быть влияние процессов хемосорбции. Для оценки вклада химической стадии в общую скорость процесса адсорбции использовали формальные уравнения, предложенные Лагергреном $(n=1)$ и Хо-Маккей $(n=2)$, имитирующие процессы массопереноса [15]. На рисунке 6 представлены результаты обработки кинетических данных по уравнениям (16) и (18) при температуре 298 К.

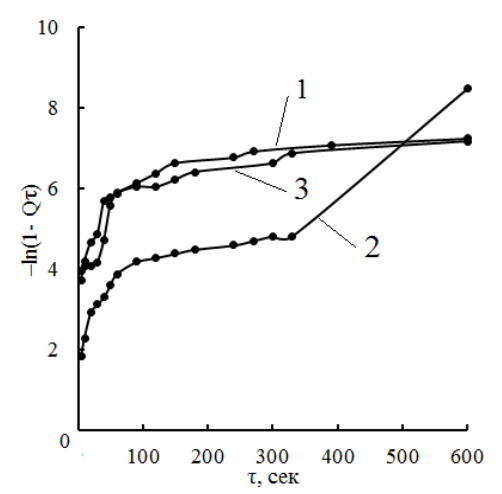

a

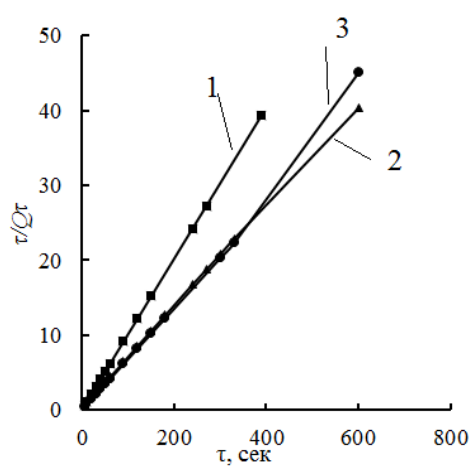

6

Рис. 6. Кинетические модели в системах « $\mathrm{Ca}^{2+}-$ сорбент»:

а) модель Лагергрена, б) модель Хо и Маккей, при температуре раствора 293 К; сорбенты: 1 - опал мергелевого типа, 2 - мергель, 3 - глина.

Как видно из рисунка 6 в системах « $\mathrm{Ca}^{2+}$ - сорбент» линеаризация уравнения прослеживается для модели Хо и Маккей. Полученные высокие коэффициенты детерминации сорбции ионов кальция в рамках модели Лагергрена (табл. 3) позволяют предположить в начальный момент времени наличие устойчивой термодинамической выгоды при осуществлении взаимодействия типа «сорбат-сорбент». Результаты, полученные после обработки, соответствующих данных представлены в табл. 3.

Таблица 3. Параметры кинетических моделей адсорбции ионов кальция на природных сорбентах $\left(\mathrm{C}_{\mathrm{Ca2}+}^{0}=1\right.$ ммоль/дм $\left.{ }^{3}\right)$

\begin{tabular}{|c|c|c|c|c|c|c|c|}
\hline \multirow[b]{2}{*}{$T, \mathrm{~K}$} & \multirow{2}{*}{$\begin{array}{c}Q_{\infty}{ }^{э к с}, \\
\text { Ммоль/Г }\end{array}$} & \multicolumn{3}{|c|}{ Модель Лагергрена $(n=1)$} & \multicolumn{3}{|c|}{ Модель Хо-Маккей $(n=2)$} \\
\hline & & $k_{l,}$ мин $^{-1}$ & $\begin{array}{l}Q_{\infty 1}{ }^{\text {pacч }}, \\
\text { ммоль/Г }\end{array}$ & $R^{2}$ & $\begin{array}{c}k_{2}, \\
\text { г/ммоль•мин }\end{array}$ & $\begin{array}{l}Q_{\infty 2}{ }^{p a c 4}, \\
\text { Ммоль/Г }\end{array}$ & $R^{2}$ \\
\hline \multicolumn{8}{|c|}{ Мергель } \\
\hline 275 & 0.211 & 0.47 & 8.08 & 0.98 & 12.8 & 0.218 & 0.99 \\
\hline 293 & 0.245 & 0.39 & 6.82 & 0.89 & 45.5 & 0.244 & 1 \\
\hline 318 & 0.250 & 0.51 & 5.77 & 0.92 & 123.4 & 0.247 & 0.99 \\
\hline \multicolumn{8}{|c|}{ Глина } \\
\hline 275 & 0.198 & 0.47 & 0.13 & 0.82 & 6.29 & 0.225 & 0.96 \\
\hline 293 & 0.250 & 0.32 & 0.002 & 0.63 & 21.83 & 0.247 & 1 \\
\hline 318 & 0.247 & 0.59 & 0.050 & 0.93 & 38.33 & 0.249 & 0.99 \\
\hline \multicolumn{8}{|c|}{ Опал мергелевого типа } \\
\hline 275 & 0.166 & 0.39 & 24.8 & 0.69 & 55.8 & 0.155 & 0.99 \\
\hline 293 & 0.166 & 0.19 & 69.1 & 0.46 & 345.3 & 0.165 & 1 \\
\hline 318 & 0.163 & 0.12 & 74.6 & 0.47 & 154.8 & 0.165 & 1 \\
\hline
\end{tabular}


Как видно из полученных данных, высокие значения коэффициентов детерминации $\left(R^{2}\right)$, сравнение экспериментальных и теоретических величин ёмкости поглощения $\left(Q_{\infty}{ }^{{ }^{\prime к с}}\right)$ ионов кальция (табл. 3$)$ позволяют судить о применимости модели Хо-Маккей (псевдо-второго порядка) для описания вклада химической стадии процесса адсорбции, а также для возможности учета межмолекулярных взаимодействий в исследуемых системах. Анализ значений констант скорости сорбции ионов кальция рассматриваемыми природными сорбентами позволяет отметить, что для модели Хо и Маккей наиболее высокие значения характерны для опала мергелевого типа. Что подтверждается малым временем установления равновесия в системе (1 мин) и возможно обусловлено цеолитной структурой породы и наличием пор различного диаметра. Более низкие значения констант скорости псевдо-второго порядка получены для мергеля, что связано со слоистой структурой породы, в которой содержание глинистой составляющей увеличивается на 10-15\%. Наиболее низкие значения рассчитаны для описания адсорбции на глине, что может быть связано со строением каолинита, имеющим микроструктуру «книжного домика» со сквозными порами размером 2-3 мкм [16].

Температурная зависимость изменения констант скорости для исследуемых сорбентов носит возрастающий характер (табл.3). Полученные закономерности согласуются с изменением коэффициентов внешней и внутренней диффузии на данных сорбентах (табл. 2). Изменение константы скорости адсорбции ионов кальция на опале мергелевого типа сопровождается конкурирующими процессами химической и физической сорбции, последняя с ростом температуры уменьшается, их суммарное проявление иллюстрируется наблюдаемым отклонением при 293К.

Для подтверждения вывода о лимитирующей стадии процесса адсорбции определены энергетические характеристики взаимодействий. Энергию активации находили аналитически и графически, используя уравнение Аррениуса в линейной форме $(13,14)$. По уравнениям Аррениуса и Эйринга рассчитали энтропию активации $\Delta S^{\#}$ (табл. 4).

Таблица 4. Энергия и энтропия активации процесса адсорбции ионов кальция на природных сорбентах

\begin{tabular}{|c|c|c|c|c|}
\hline \multirow{2}{*}{ Сорбент } & \multirow{2}{*}{$E_{a}$, кДж/моль } & \multicolumn{3}{|c|}{$\Delta S^{\#}$, Дж/моль $\cdot \mathrm{K}$} \\
\cline { 3 - 5 } & & $275 \mathrm{~K}$ & $293 \mathrm{~K}$ & $318 \mathrm{~K}$ \\
\hline Мергель & 38 & -123 & -123 & -122 \\
\hline Глина & 31 & -535 & -535 & -536 \\
\hline Опал мергелевого типа & 22 & -305 & -306 & -307 \\
\hline
\end{tabular}

Исходя из значений изменения величины энергии активации (табл. 4), находящихся в пределах от 20 до 40 кДж/моль, для данных природных сорбентов можно предположить, что скорость - лимитирующей стадией процесса адсорбции ионов кальция является химическое взаимодействие.

Отрицательные значения энтропии активации процесса адсорбции свидетельствуют о благоприятной ориентации сорбата и активных центров поверхности сорбента, что свидетельствует об образовании промежуточного активированного комплекса (интермедиата) в системе «сорбат - растворитель-сорбент», а также подтверждает значительный вклад хемосорбции в механизм процесса адсорбции.

\section{Заключение}

Анализ изотерм адсорбции при исследуемых температурах, термодинамические параметры, изменение коэффициентов диффузии свидетельствуют о характере 
процесса адсорбции ионов кальция: на глине и мергеле - эндотермический; на опале мергелевого типа - экзотермический. Характер изотерм адсорбции и изменение значений изостерических теплот позволяют охарактеризовать сорбенты как разнопористые материалы.

Экспериментальные данные, полученные в результате исследования кинетики адсорбции ионов кальция в статических условиях, а также проведение математической обработки интегральных кинетических кривых, позволяют установить факт аппроксимации уравнений диффузионной и формальной кинетики и дают основание считать, что, в целом, процесс адсорбции ионов кальция включает в себя диффузионный режим, но наибольший вклад вносит кинетический режим, который в зависимости от природы сорбента определяется смешанодиффузионным механизмом. Обе стадии (и «пленочная»- внешнедиффузионная и «гелевая»- внутридиффузионная) оказывают влияние на скорость кинетической стадии: в начальном интервале времени до одной минуты преобладает внешнедиффузионный механизм, после - внутридиффузионный.

Установлено, что при адсорбции ионов кальция на исследуемых природных сорбентах лимитирующей является кинетическая стадия, представляющая собой комбинацию внешней и внутренней диффузии, с некоторым преобладанием гелевого механизма диффузии для мергеля и глины, пленочного для опала мергелевого типа, и описывается моделью Хо-Маккей $(n=2)$, учитывающий вклад хемосорбции.

Анализируя в целом полученные результаты можно с большей долей вероятности предположить образование в системе «сорбент-растворитель-сорбат» интермедиатов или ассоциатов. Об ассоциативном механизме процесса свидетельствуют полученные кинетические данные - кинетическое уравнение второго порядка, низкие значения энергии активации процесса диффузии и сильно отрицательные значения энтропии активации, величины изостерических теплот.

Экспериментально найденные количественные характеристики адсорбции ионов кальция, позволяют сделать вывод о возможности применения изучаемых сорбентов в качестве носителей для производства фрикционных противогололедных материалов [21]. Процесс работы полученных противогололедных систем происходит за счет выделения энергии при совместном физико-химическим взаимодействии носителей и ионов кальция с поверхностью льда.

\section{Список литературы}

1. Lv J., Song Y., Jiang L., Wang J. // American Chemical Society. 2014. Vol. 8(4). pp. 3152-3169. DOI:10.1021/nn406522n.

2. Farnam Y., Dick S., Wiese A., Davis J. et al. // Cement and Concrete Composites. 2015. Vol. 64. pp. 1-15.

3. Алыков Н.М., Сютова Е.А. // Экологические системы и приборы. 2007. № 8. C. 46-48.

4. Сютова Е.А., Алыков Н.М. // Экология и промышиленность России. 2012. № 2. С. 4751.

5. Громалова В.О., Федотов А.И., Зедгенизов В.Г., Гергенов С.М. // Вестник Сибирского автомобильно-дорожного университета. 2018. T. 15. № 1 (59). С. 55-60.

6. Джигола Л.А., Садомцева О.С., Шакирова В.В., Каргина К.В. и др. // Известия высших учебных заведений. Серия: Химия и химическая технология. 2018. Т. 61. № 9-10. С. 105-112.

7. Джигола Л.А., Сютова Е.А. // Известия высших учебных заведений. Серия: Химия и химическая технология. 2018. Т. 61. № 9-10. C. 98-104.

8. Sirotkina E. E., Novoselova L. YU. // Chemistry for Sustainable Development. 2005. Vol. 13. pp. 359-375. 
9. Дударев В.И., Иринчинова Н.В., Филатова Е.Г. // Изв. вузов. Химия и хим. технология. 2017. Т. 60. № 1. С. 75-80.

10. Tataeva S.D., Ramazanov A.Sh., Magomedov K.E., Bakhmudova A.G. // Journal of Analytical Chemistry. 2014. Vol. 69(1). pp. 4550.

11. Бойд Д.Е., Адамсон А.В., Майерс Л.С. Хроматография. М. Изд-во иностр. лит-ры, $1949.333 \mathrm{c}$.

12. Рамазанов А.Ш., Есмаил Г.К. // Вестник Дагестанского государственного универсиmema. 2014. T. 1. C.179-183.

13. Низамова Г.Р., Галимова Р.З., Шайхиев И.Г. // Вестник технологического универсиmema. 2017. Т. 20. № 11. С. 142-148.

14. Киекпаев М.А., Строева Э.В. // Вестник ОГУ. 2006. № 5. С. 35-39.
15. Пимнева Л.А., Нестерова Е. Л. // Вестник омского университета. 2011. № 2. С. 130-134.

16. Брилинг И.А. Фильтрация в глинистых nородаx. М. ВИЭМС. 1984. $61 \mathrm{c.}$

17. Bleza M.A., Figliolia N.M., Maroto A.J.G., Regazzoni A.E. // J. of Colloid and Int. Sci. 1984. Vol. 101(2). pp. 410-418.

18. Макаров А.В. Дисс. канд. техн. наук. Томск. 2013. $154 \mathrm{c}$.

19. Hawari A., Rawajfih Z., Nsour N. // J. Hazard. Mater. 2009. Vol. 168. pp. 1284-1289

20. Алосманов Р.М. // Вестн. Моск. ун-та. Сер.2. Химия. 2011. Т. 52. № 2. С. 145-148.

21. Алыков Н.М., Сютова Е.А., Алыков Е.Н. Патент РФ. № 2378311. 2007.

\title{
Research of the kinetic regularities of calcium ions solid-phase concentration by natural sorbents
}

\author{
Syutova Elizaveta A. ${ }^{1}$, Dzhigola Lyudmila A. ${ }^{2}$ \\ ${ }^{I}$ Federal State Budgetary Educational Institution of Higher Education \\ "Astrakhan State Medical University", Astrakhan \\ ${ }^{2}$ Federal State Budgetary Educational Institution of Higher Education \\ "Astrakhan State University", Astrakhan
}

The most common way to prevent slippy roads in winter is by using de-icing mixtures, which combine salts and organic materials and thus have both chemical and mechanical effects. Such mixtures are usually obtained through sorption saturation with various modifying components, most commonly sodium, potassium, calcium, and magnesium chlorides. The aim of this study is to determine the optimal conditions for the adsorption of calcium ions by natural sorbents in the Astrakhan Region, as well as to evaluate the approximations of chemical and diffusion models describing the kinetics of sorption concentration in order to create anti-icing mixtures.

The sorbents: marl, an opal mineral of marl type (silica clay), and clay, were obtained by the nonchemical processing of natural raw materials of the Astrakhan region, extracted by open pit mining.

The kinetic analysis of the adsorption of calcium ions included evaluating the loss of sorbate at different time intervals. The evaluation was performed by means of direct potentiometry using an ELIS-121Ca ion-selective electrode with regard to a silver chloride reference electrode EVL-1M3.1, taking into account the background electrolyte. The effect of the medium on the adsorption process was studied using an ESK10601/7 combination glass electrode. It was determined that the silica clay reaches maximum adsorption capacity for calcium ions at $\mathrm{pH}=6.94$, marl at $\mathrm{pH}=5.61$, and clay at $\mathrm{pH}=6.45$.

The analysis of the $\mathrm{Ca}^{2+}$ ions adsorption isotherms at the studied temperatures allows us to determine the nature of the process: it is endothermic on clay and marl, and exothermic on silica clay. Silica clay has the maximum sorption capacity for $\mathrm{Ca}^{2+}$ ions; for clay and marl these values are practically the same. To identify the sorbate interactions, the isosteric heats of adsorption of $\mathrm{Ca}^{2+}$ ions were calculated. The analysis of the nature of the changein the isosteric heats of adsorption at various values of the degree of filling of the sorbent indicates that the studied natural sorbents have heterogeneous surfaces with centres of varying degrees of activity.

The interpretation of the kinetics of adsorption of calcium ions on natural sorbents allows us to state that the adsorption process of calcium ions proceeds in a mixed diffusion mode. Graphical determination and theoretical calculation of the constants of external and internal diffusion, the values of the Bio-Rad's criterion, and the apparent activation energy lead to the conclusion that for marl and clay the internal diffusion has the strongest effect at the limiting stage, while for silica clay it is external diffusion. The chemical interaction of the sorbate ions with the surface of sorbents contributes to the overall rate of the process. This is 
confirmed by the fact that the experimental data corresponds to the kinetic model proposed by Ho and McKay.

The quantitative characteristics of adsorption of calcium ions determined by the experiment allow us to conclude that it is possible to use the studied sorbents as carriers for the production of friction de-icing agents. The obtained de-icing systems become active due to the release of energy during the joint physicochemical interaction of carriers and calcium ions with the ice surface.

Keywords: sorption concentration, chemisorption, isotherms, internal diffusion, external diffusion, mineral sorbents

\section{References}

1. Lv J., Song Y., Jiang L., Wang J., American Chemical Society, 2014, Vol. 8(4), pp. 3152-3169. DOI:10.1021/nn406522n.

2. Farnam Y., Dick S., Wiese A., Davis J., Bentz D., \& Weiss J., Cement and Concrete Composites, 2015, Vol. 64, pp. 1-15.

3. Alykov N.M., Syutova E.A., Ekologicheskie sistemy $i$ pribory, 2007, No 8, pp. 4648.

4. Syutova E.A., Alykov N.M., Ekologiya $i$ promyshlennost' Rossii, 2012, No 2, pp. 47-51.

5. Gromalova V.O., Fedotov A.I., Zedgenizov V.G., Gergenov S.M., Vestnik Sibirskogo gosudarstvennogo avtomobil'no-dorozhnogo universiteta, 2018, Vol. 15, No 1 (59), pp. 55-60.

6. Dzhigola L.A., Sadomtseva O.S., Shakirova V.V., Kargina K.V. et al., Izvestiya vysshikh uchebnykh zavedenii. Seriya: Khimiya i khimicheskaya tekhnologiya, 2018, Vol. 61, No 9-10, pp. 105-112.

7. Dzhigola L.A., Syutova E.A., Izvestiya vysshikh uchebnykh zavedenii. Seriya: Khimiya i khimicheskaya tekhnologiya, 2018, Vol. 61, No 9-10, pp. 98-104.

8. Sirotkina E.E., Novoselova L.Yu., Chemistry for Sustainable Development, 2005, Vol. 13, pp. 359-375.

9. Dudarev V.I., Irinchinova N.V., Filatova E.G., Izv. vuzov. Khimiya i khim. Tekhnologiya, 2017, Vol. 60, No 1, pp. 7580.

Джигола Людмила Александровна - к.х.н., доцент, заведующая кафедрой аналитической и физической химии, ФГБОУ ВО «Астраханский государственный университет», Астрахань

Сютова Елизавета Анатольевна - старший преподаватель кафедры химии фармацевтического факультета, ФГБОУ ВО Астраханский государственный медицинский университет, Астрахань
10. Tataeva S.D., Ramazanov A.Sh., Magomedov K.E., Bakhmudova A.G. Journal of Analytical Chemistry, 2014, Vol. 69(1), pp. 45-50.

11. Boid D.E., Adamson A.V., Maiers L.S., Khromatografiya, M., Izd-vo inostr. lit-ry, 1949, 333 p.

12. Ramazanov A.Sh., Esmail G.K., Vestnik Dagestanskogo gosudarstvennogo universiteta, 2014, Vol. 1, pp. 179-183.

13. Nizamova G.R., Galimova R.Z., Shaikhiev I.G., Vestnik tekhnologicheskogo universiteta, 2017, Vol. 20, No 11, pp. 142-148.

14. Kiekpaev M.A., Stroeva E.V., Vestnik $O G U, 2006$, No 5, pp. 35-39.

15. Pimneva L.A., Nesterova E.L., Vestnik omskogo universiteta, 2011, No 2, pp. 130-134.

16. Briling I.A., Fil'tratsiya v glinistykh porodakh, M., VIEMS, 1984, 61 p.

17. Bleza M.A., Figliolia N.M., Maroto A.J.G., Regazzoni A.E., J. of Colloid and Int. Sci., 1984, Vol. 101(2), pp. 410-418.

18. Makarov A.V., Diss. kand. tekhn. nauk, Tomsk, 2013, 154 p.

19. Hawari A., Rawajfih Z., Nsour N., J. Hazard. Mater., 2009, Vol. 168, pp. 1284-1289.

10. Alosmanov R.M., Vestn. Mosk. un-ta. Ser.2. Khimiya, 2011, Vol. 52, No 2, pp. 145148.

21. Alykov N.M., Syutova E.A., Alykov E.N. Patent RF, No 2378311, 2007.

Dzhigola Lyudmila A. - Ph. D (Chemistry), associate professor, head of the department of Analytical and Physical Chemistry, Astrakhan State University, Astrakhan', Russia, djegola@mail.ru

Syutova Elizaveta A. - Senior lecturer of the department of Chemistry of the Pharmaceutical Faculty, Astrakhan State Medical University, Astrakhan, Russia, elizoveta_ast@mail.ru 